\title{
Finite Element Vibration Analysis of Cylindrical Shells Conveying Fluid with Considering Acoustic-Structure Interactions*
}

\author{
Weui Bong JEONG**, Young Soo SEO**, Se Jin AHN** and Wan Suk YOO**
}

\begin{abstract}
The dynamic behavior of the cylindrical shell with uniform flow is formulated by the finite element method. The dynamics of the shell is based on Donnell's theory and the fluid in cylindrical shell is considered satisfying the Helmholtz equation. The effective thickness of fluid is calculated according to the circumferential modes and the frequencies. An estimation of the FRF (frequency response function) of the shell with taking into consideration of the coupled effects of the internal fluid is presented. These results are compared with the results considering fluid satisfying Laplace equation. The influence of fluid velocity on the FRF is also discussed.
\end{abstract}

Key Words: Cylindrical Shells, Fluid Flow, Finite Element Analysis, Acoustic-Structure Interactions

\section{Introduction}

The dynamic behavior of the cylindrical shell with fluid flow has been studied for a long time. There were many researches to analyze the pipe conveying fluid. The finite element method was used widely to study the behavior of the shell. If the beam element is used for the finite element formulation, the fluid in the shell could only have an effect of added mass on the shell. The shell element can take into consideration of the interactions between the shell and fluid. However the shell element needs lots of nodes and elements in a model, which takes many times to analyze. Paidoussis and Issid ${ }^{(1)}$ used the beam element to discuss the dynamics and stability of pipes conveying fluid with various boundary conditions. Ginsberg ${ }^{(2)}$ used beam element to carry out the stability analysis, based on the Floquet theory, of the pipe with a pulsating flow. Chen and Rosenberg ${ }^{(3)}$ presented frequency spectra of symmetric and asymmetric modes, and discussed the effects of fluid velocity and other parameters. Mazuch et al. ${ }^{(4)}$ studied the natural frequencies and the mode shapes using the shell theory in contact with inviscid, incompressible fluid by finite element method and experiment. Zhang et al. ${ }^{(5)}$ studied the coupled structural-acoustic analysis of

* Received 25th February, 2005 (No. 05-5023)

** Department of Mechanical Engineering, Pusan National University, Jangjeon-dong, Kumjung-ku, Pusan 609-735, South Korea. E-mail: wbjeong@pusan.ac.kr fluid-filled cylindrical shells using the wave propagation method. The natural frequencies by the wave propagation method were compared with those by finite element method and boundary element method. Zhang et al. ${ }^{(6)}$ presented the finite element formulation of the thin shell conveying fluid by applying Sanders' thin shell theory. The fluid in the shell was considered as incompressible and inviscid, whose behavior satisfies Laplace equation. Most of previous works were only interested in the natural frequencies or the stability analysis. Seo et al. ${ }^{(7)}$ presented the estimation of frequency response characteristics of forced vibration of the cylindrical shell conveying fluid. But the fluid in the shell was assumed to satisfy Laplace equation, which means that the coupled effect of the fluid is constant regardless of the frequency. The Laplace equation is a special case considering the frequency of $0 \mathrm{~Hz}$ in Helmholtz equation. Therefore, using Laplace equation is not desirable in acoustic analysis of higher frequency range.

In this paper, the cylindrical shell was coupled with the internal fluid satisfying the Helmholtz equation with uniform flow. The dynamic behavior of cylindrical shell is assumed to satisfy the Donnell' shell theory. The dynamic pressure acting on the shell wall is derived. The characteristics of the shell with internal fluid satisfying Helmholtz equation are compared with the results of the fluid satisfying Laplace equation. The effective thickness of fluid according to the circumferential mode and the frequency 
is presented. The effect of the velocity of uniform flow is also discussed.

\section{Finite Element Formulation}

The shell element type could be usually used to formulate the cylindrical shell. But, it causes many elements in the shell model and much time to analyze. The circumferential modes of the shell are assumed to be known to overcome this problem. Generally, the circumferential mode of the shell is known as sinusoidal function. Figure 1 shows a model of cylindrical shell conveying internal fluid. The displacement of a cylindrical shell can be assumed by the following Fourier's cosine expansion ${ }^{(8)}$ :

$$
\{\vec{u}\}=\left\{u_{x} \cos n \theta, u_{\theta} \sin n \theta, u_{r} \cos n \theta\right\}^{T},
$$

where $n$ is the circumferential mode number and $u_{x}, u_{\theta}$ and $u_{r}$ are the displacements of shell in axial, tangential and radial direction. Figure 2 shows the circumferential mode shapes according to the circumferential mode number $(n)$. This assumption makes it possible not to generate meshes in circumferential direction, which gives the reduction of degree-of-freedom in finite element formulation. Seo et al. ${ }^{(7)}$ called this type of element as beam-like shell element. The mesh generation of beam-like shell element is the same as that of beam element. For the finite element formulation, the displacements in elements should be expressed by the shape function and nodal displacements, which gives the followings:

$$
\{u\}=\left\{\begin{array}{lll}
u_{x} & u_{\theta} & u_{r}
\end{array}\right\}^{T}=\left[N_{s}\right]\{\bar{u}\},
$$

where

$$
\{\bar{u}\}=\left\{\begin{array}{llllllll}
\bar{u}_{x i} & \bar{u}_{\theta i} & \bar{u}_{r i} & \phi_{i} & \bar{u}_{x j} & \bar{u}_{\theta j} & \bar{u}_{r j} & \phi_{j}
\end{array}\right\}^{T} .
$$

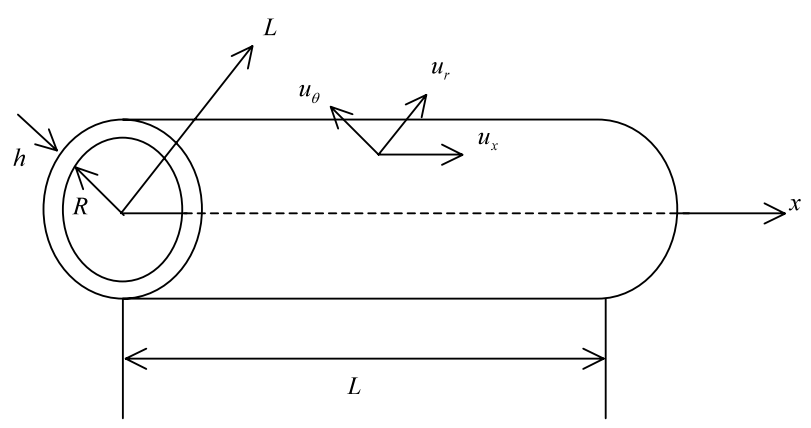

Fig. 1 A model of cylindrical shell conveying internal fluid

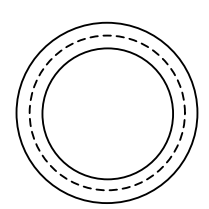

$n=0$

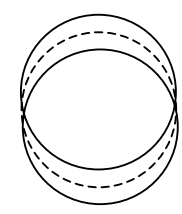

$n=1$

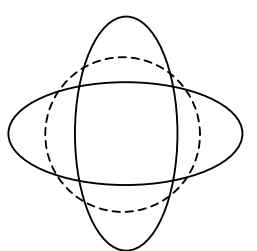

$n=2$
Fig. 2 Circumferential mode shapes according to the circumferential mode number $(n)$
Here, $\left[N_{s}\right]$ is the shape function matrix and $\{\bar{u}\}$ is a displacement vector at nodal points. Any shape function can be defined by user. Linear functions are used as shape functions in this paper. The radial displacement of cylindrical shell can be assumed to behave like a lateral displacement of a beam. Thus, the shape function in Eq. (2) is the same as that of lateral vibration of a beam. The $\phi=\partial u_{r} / \partial x$ denotes the slope of $u_{r}$. The mass, the stiffness matrix and the force vector of the cylindrical structure can be given as follows:

$$
\begin{aligned}
{[m]_{s} } & =\int_{A_{s}} \rho_{s} h\left[N_{s}\right]^{T}\left[N_{s}\right] d A_{s} \\
& =\rho_{s} h R \int_{0}^{2 \pi} \int_{0}^{l_{e}}\left[N_{s}\right]^{T}\left[N_{s}\right] d x d \theta, \\
{[k]_{s} } & =\int_{A_{s}}[B]^{T}[D][B] d A_{s} \\
& =R \int_{0}^{2 \pi} \int_{0}^{l_{e}}[B]^{T}[D][B] d x d \theta, \\
\{f\}_{s} & =\int_{A_{s}}\left[N_{s r}\right]^{T} p_{a} d A_{s}+\int_{A_{s}}\left[N_{s}\right]^{T} q_{s} d A_{s},
\end{aligned}
$$

where $l_{e}$ is a length of shell element, $\rho_{s}$ is the density, $h$ is the thickness, $R$ is the radius of the shell, $[B]$ is the matrix which denotes the strain-displacement relation, $[D]$ is the matrix of material constants which denotes the stressstrain relations and $\left[N_{s r}\right]$ is the shape function of the radial component. The first term in Eq. (6) represents the coupling effect of the fluid. The acoustic pressure $p_{a}$ of the fluid at the interface acts normally on the structure of cylindrical shell. The second term of Eq. (6) represents the equivalent force due to external distributed force $\left(q_{s}\right)$.

To consider the effects of the fluid in the cylindrical shell, let us assume that the fluid satisfies the acoustic wave equation with uniform flow. The equation of motion of the fluid can be written in the cylindrical coordinate system as follows ${ }^{(3)}$ :

$$
\nabla^{2} \phi-\frac{1}{c_{f}^{2}}\left(\frac{\partial^{2} \phi}{\partial t^{2}}+2 U \frac{\partial^{2} \phi}{\partial x \partial t}+U^{2} \frac{\partial^{2} \phi}{\partial x^{2}}\right)=0
$$

where $\phi$ is the velocity potential, $c_{f}$ is the wave speed of the fluid and $U$ is the mean flow velocity to axial direction. The displacements of the shell can also be expressed in the form of wave propagation, associated with an axial wave number $k_{x}$ and a circumferential modal parameter $n$.

$$
\begin{aligned}
& u_{x}=\bar{u}_{x} \cos (n \theta) e^{j\left(\omega t-k_{x} x\right)}, \\
& u_{\theta}=\bar{u}_{\theta} \sin (n \theta) e^{j\left(\omega t-k_{x} x\right)}, \\
& u_{r}=\bar{u}_{r} \cos (n \theta) e^{j\left(\omega t-k_{x} x\right)},
\end{aligned}
$$

where $\bar{u}_{x}, \bar{u}_{\theta}$ and $\bar{u}_{r}$ are the wave amplitudes in the $x, \theta$, $r$ directions and $\omega$ is the circular driving frequency. The radial velocity of the shell and fluid at the interface $r=$ $R$ must be same. This gives the following compatibility condition: 


$$
\left.\frac{\partial \phi}{\partial r}\right|_{r=R}=\frac{\partial u_{r}}{\partial t}+\left.U \frac{\partial u_{r}}{\partial x}\right|_{r=R} .
$$

To solve the wave equation, let us assume the velocity potential $\phi$ as follows:

$$
\phi=\bar{\phi}(r) \cos n \theta e^{j\left(\omega t-k_{x} x\right)} .
$$

Substituting Eq. (12) into Eq. (7) and taking into consideration of the compatibility condition given in Eq. (11) leads the solution as follows:

$$
\begin{aligned}
\phi & =\frac{R F_{n}\left(k_{r} R\right)}{k_{r} R F_{n, r}\left(k_{r} R\right)}\left(\frac{\partial u_{r}}{\partial t}+U \frac{\partial u_{r}}{\partial x}\right) \\
& =j k_{x}\left(c_{p}-U\right) R \frac{F_{n}\left(k_{r} R\right)}{k_{r} R F_{n, r}\left(k_{r} R\right)} \bar{u}_{r} \cos n \theta e^{j\left(\omega t-k_{x} x\right)},
\end{aligned}
$$

if $\frac{U}{c_{f}}\left(1-\frac{c_{p}}{U}\right)>1$

$$
F_{n}\left(k_{r} r\right)=J_{n}\left(k_{r} r\right), \quad k_{r}=k_{x}\left[\frac{U^{2}}{c_{f}^{2}}\left(1-\frac{c_{p}}{U}\right)^{2}-1\right]^{1 / 2},(1
$$

if $\frac{U}{c_{f}}\left(1-\frac{c_{p}}{U}\right)<1$

$$
F_{n}\left(k_{r} r\right)=I_{n}\left(k_{r} r\right), \quad k_{r}=k_{x}\left[1-\frac{U^{2}}{c_{f}^{2}}\left(\frac{c_{p}}{U}-1\right)^{2}\right]^{1 / 2},
$$

where $c_{p}$ is the wave speed of the shell, $J_{n}$ is the Bessel function of the first kind of order $n$ and $I_{n}$ is the modified Bessel function of the first kind of order $n$.

The acoustic pressure at the interface of fluid and shell can be expressed as follow:

$$
\begin{aligned}
& p_{a}(x, \theta, R, t) \\
& \quad=-\left.\rho_{f}\left(\frac{\partial \phi}{\partial t}+U \frac{\partial \phi}{\partial x}\right)\right|_{r=R} \\
& =\rho_{f} R k_{x}^{2} c_{p}^{2}\left(1-\frac{U}{c_{p}}\right)^{2} \frac{F_{n}\left(\kappa_{r}\right)}{k_{r} R F_{n, r}\left(k_{r} R\right)} \bar{u}_{r} \cos n \theta e^{j\left(\omega t-k_{x} x\right)}
\end{aligned}
$$

Substituting Eqs. (8)-(10), (16) into the equation of the motion of the shell ${ }^{(9)}$, the equation of motion of the coupled system taking into account the acoustic pressure of the internal fluid can be written in matrix form as

$$
\left[\begin{array}{lll}
C_{11} & C_{12} & C_{13} \\
C_{12} & C_{22} & C_{23} \\
C_{13} & C_{23} & C_{33}
\end{array}\right]\left\{\begin{array}{l}
\bar{u}_{x} \\
\bar{u}_{\theta} \\
\bar{u}_{r}
\end{array}\right\}=\left\{\begin{array}{l}
0 \\
0 \\
0
\end{array}\right\},
$$

where

$$
\begin{aligned}
C_{11}= & -k_{x}^{2}-\frac{n^{2}}{2 R^{2}}(1-v)+\frac{\omega^{2}}{c_{p}^{2}}, \quad C_{12}=-j \frac{n}{2 R}(1+v) k_{x}, \\
C_{13}= & j \frac{v}{R} k_{x}, \\
C_{22}= & \frac{1}{2}(1-v) k_{x}^{2}+\frac{n^{2}}{R^{2}}-\frac{\omega^{2}}{c_{p}^{2}}, \quad C_{23}=-\frac{n}{R^{2}}, \\
C_{33}= & -\frac{1}{R^{2}}+\beta^{2}\left(k_{x}^{4} R^{2}+2 k_{x}^{2} n^{2}+\frac{n^{4}}{R^{2}}\right) \\
& -\frac{\rho_{f}}{\rho_{s}} \frac{R}{h} k_{x}^{2}\left(1-\frac{U}{c_{p}}\right)^{2} \frac{F_{n}\left(k_{r} R\right)}{k_{r} R F_{n, r}\left(k_{r} R\right)}-\frac{\omega^{2}}{c_{p}^{2}} .
\end{aligned}
$$

Here, $v$ is the poisson's ratio.

For a nontrivial solution of these equations, the determinant of the coefficients of Eq. (17) should be zero. It is notable that the following characteristic equation is the frequency-dependant equation.

$$
\left|C_{i j}\right|=0 . \quad(i, j=1,2,3)
$$

This equation is not a polynomial function but a complex transcendental function. The axial acoustic wave number $k_{x}$ can be determined as the solution of Eq. (18). The value of $k_{x}$ is the function of frequency $\omega$. Obtaining $k_{x}$ from Eq. (18), the radial acoustic wave number $k_{r}$ can be calculated as follows:

$$
k_{r}{ }^{2}=\left(\frac{c_{p}}{c_{f}}\right)^{2}\left(1-\frac{U}{c_{p}}\right)^{2}\left(\omega / c_{p}\right)^{2}-k_{x}{ }^{2} .
$$

Substituting the acoustic pressure given in Eq. (16) into Eq. (6) gives the following coupled equation of motion.

$$
\left([m]_{s}+[m]_{f}\right)\{\ddot{\bar{u}}\}+[c]_{f}\{\dot{\bar{u}}\}+\left([k]_{s}+[k]_{f}\right)\{\bar{u}\}=\{f\}_{s},
$$

where,

$$
\begin{aligned}
& {[m]_{f}=\int_{A_{f}} \rho_{f} N_{s r}^{T} N_{s r} h_{f} d A_{f},} \\
& {[c]_{f}=2 U \int_{A_{f}} N_{s r}{ }^{T} \frac{\partial N_{s r}}{\partial x} h_{f} d A_{f},} \\
& {[k]_{f}=-U^{2} \int_{A_{f}} \frac{\partial N_{s r}^{T}}{\partial x} \frac{\partial N_{s r}}{\partial x} h_{f} d A_{f},} \\
& h_{f}=\operatorname{Re}\left(\frac{R F_{n}\left(k_{r} R\right)}{k_{r} R F_{n, r}\left(k_{r} R\right)}\right) .
\end{aligned}
$$

Here, $h_{f}$ shows the effective thickness of fluid, which depends on the order of circumferential mode and the frequency. Therefore, the mass, damping, stiffness matrices are also the function of the order of circumferential mode and the frequency. But in the previous study ${ }^{(7)}$, the flow in the shell is assumed to satisfy the Laplace equation instead of Eq. (7) in this paper. It means that the effective thickness of fluid is constant regardless of the frequency, which is given as follows ${ }^{(7)}$ :

$$
h_{f}=\operatorname{Re}\left(\frac{J_{n}(\lambda R)}{\partial J_{n}(\lambda R) / \partial r}\right), \quad n=1,2, \cdots
$$

where characteristic values, $\lambda$, can be obtained from the characteristic equation given by Zhang et al. ${ }^{(6)}$ It is function of only circumferential mode. In case of obtaining the FRF according to the circumferential mode it has an affect on the mass, damping and stiffness matrix as constant value. In this paper, however, the fluid is assumed to satisfy the wave equation given in Eq. (7), and the effective thickness of the fluid varies not only by the circumferential modes but also by the driving frequencies. Therefore, the mass, damping and stiffness matrices are also functions of the circumferential modes and frequencies. 


\section{Frequency Response}

The axial acoustic wave number, $k_{x}$, determined from Eq. (18), depends on the order, $n$ of circumferential mode and frequency. The effective thickness of fluid, $h_{f}$ given in Eq. (24), depends on the characteristic value $k_{r}$. Thus, the effective thickness $h_{f}$ must be calculated according to the order $n$ and frequency. The coupled equation of motion of a cylindrical shell conveying fluid, given in Eq. (20), must be solved according to the order, $n$ of circumferential mode and frequency.

Let us assume the external harmonic force applied to the structure of cylindrical shell as

$$
\{f\}_{s}=\{\bar{f}\} e^{j \omega t} .
$$

The harmonic response $\{\bar{u}\}$ in Eq. (20) can be determined as the solution of the following algebraic equation

$$
\left[\left([k]_{s}+[k]_{f}\right)-\omega^{2}\left([m]_{s}+[m]_{f}\right)+j \omega[c]_{f}\right]\{\bar{u}\}=\{\bar{f}\}_{s} .
$$

Since equations of motion are given by the superposition of the solution according to order, $n$. The displacement of a cylindrical shell can be obtained as follows:

$$
\begin{aligned}
& u_{x}=\sum_{n=0}^{\infty} \bar{u}_{x, n} \cos n \theta e^{j\left(\omega t-k_{x, n} x\right)}, \\
& u_{\theta}=\sum_{n=1}^{\infty} \bar{u}_{\theta, n} \sin n \theta e^{j\left(\omega t-k_{x, n} x\right)}, \\
& u_{r}=\sum_{n=0}^{\infty} \bar{u}_{r, n} \cos n \theta e^{j\left(\omega t-k_{x, n} x\right)} .
\end{aligned}
$$

The FRF can be estimated from Eq. (27) by setting external force equal to unity.

\section{Numerical Examples}

Several calculations are carried out to validate the presented method. The test model shown in Fig. 3 has following dimensions: length $(L)=0.231[\mathrm{~m}]$, radius $(R)=$ $0.07725[\mathrm{~m}]$, thickness $(h)=0.0015[\mathrm{~m}]$, Young's Modulus $(E)=2.05 \times 10^{11}\left[\mathrm{~N} / \mathrm{m}^{2}\right]$, Poisson's ratio $(v)=0.3$, density of steel $\left(\rho_{s}\right)=7800\left[\mathrm{~kg} / \mathrm{m}^{3}\right]$, density of fluid $\left(\rho_{f}\right)=$ $1000\left[\mathrm{~kg} / \mathrm{m}^{3}\right]$, wave speed of steel $\left(c_{p}\right)=5000[\mathrm{~m} / \mathrm{s}]$, wave speed of water $\left(c_{f}\right)=1500[\mathrm{~m} / \mathrm{s}]$. The boundary condition is clamped-free.

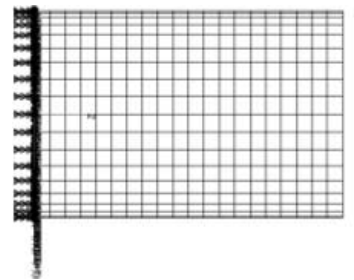

(a) Nastran (shell element)

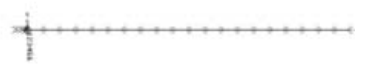

(b) Presented model (beamlike shell element)
Fig. 3 Element Type used in Numerical example
Table 1 shows the number of nodes and degreesof-freedom used for the analysis. The cylindrical shell was divided equally into 20 elements in the axial direction for both Nastran (shell element) model and the presented (beam-like-shell element) model. The shellelement model needed additional division into 36 elements in circumferential direction, while the presented model did not need any more division. The number of degree-of-freedom by the presented method was further less than that by Nastran. Instead, the presented method must solve the equations of motion for every order of circumferential mode, $n=1,2, \cdots$.

In the first calculation, the fluid-filled cylindrical shell is performed. Figure 4 shows the dependence of the axial wave number on the frequency. It is notable that the axial wave number varied according to both the frequency and the circumferential mode. The cut-on frequencies of the axial mode increase according to the circumferential modes.

Figure 5 shows the dependence of effective thickness on the frequency. In Ref. (7), when the fluid is assumed to satisfy Laplace equation, the effective thickness becomes constant as shown in Table 2, which is a special case of

Table 1 Comparison of the number of D.O.F used for the analysis

\begin{tabular}{|c|c|c|}
\hline & Nastran & Presented \\
(shell element) & \\
\hline The number of circumferential node & 36 & - \\
\hline The number of axial node & 21 & 21 \\
\hline The number of total node & 756 & 4 \\
\hline D.O.F per node & 5 & 48 \\
\hline Total D.O.F of model & 3780 & \\
\hline
\end{tabular}

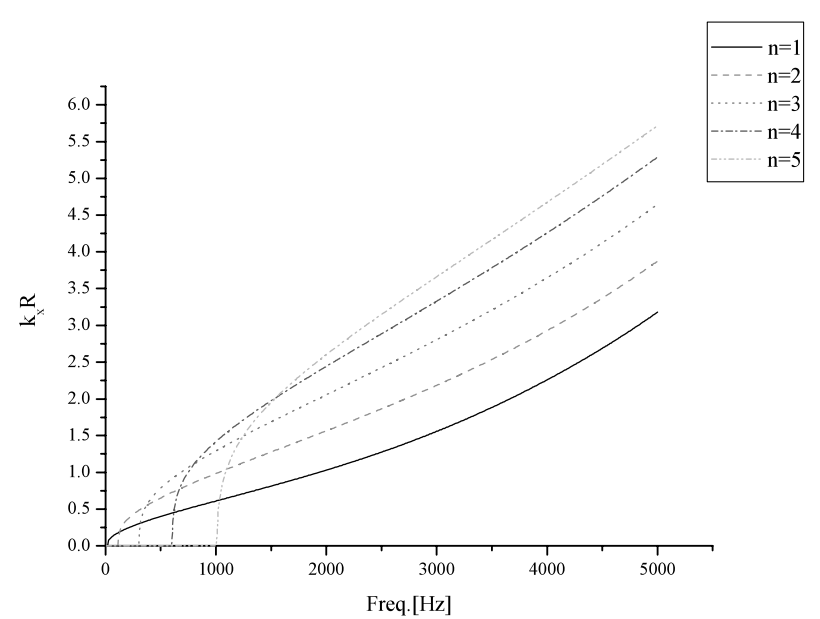

Fig. 4 The values of the axial wave number for circumferential mode $(n)$ 


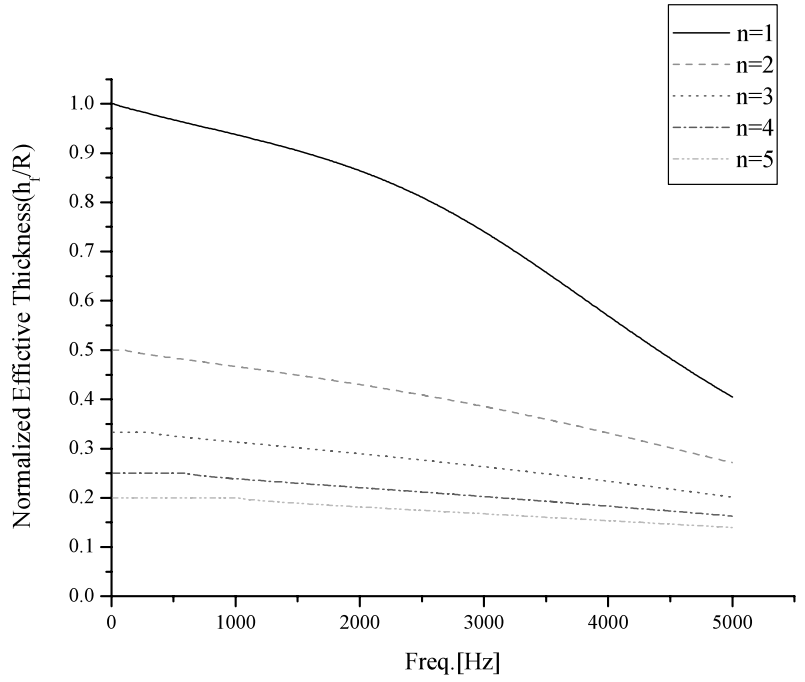

Fig. 5 Effective thickness of the internal fluid for circumferential mode $(n)$

Table 2 Effective thickness of the internal fluid satisfying Laplace equation $^{(7)}$

\begin{tabular}{|c|c|}
\hline $\begin{array}{c}\text { Order of } \\
\text { circumferential Mode } \\
(n)\end{array}$ & $\begin{array}{c}\text { Non-dimensional effective } \\
\text { thickness }\left(h_{f} / R\right)\end{array}$ \\
\hline 1 & 1.000 \\
\hline 2 & 0.500 \\
\hline 3 & 0.333 \\
\hline 4 & 0.251 \\
\hline 5 & 0.202 \\
\hline
\end{tabular}

$0 \mathrm{~Hz}$ in Fig. 5. Therefore, using Laplace equation is not desirable in acoustic analysis of higher frequency range. But in the presented model, the effective thickness varies according to both the circumferential mode and the frequency. The effective thickness of the first circumferential mode decrease rapidly according to the frequency. But the effective thickness of the higher circumferential mode decrease slowly.

In other to calculate the FRF, an external force in radial direction, $F_{r}$, was assumed to apply at the free end of the cylindrical shell. The receptance (displacement/force) was obtained at the driving point. The difference of the results between the previous method ${ }^{(7)}$ and the presented method is shown in Fig. 6. The previous method assumed that the fluid satisfies Laplace equation, but the presented method assumed the fluid satisfies Helmholtz equation. The difference comes from the reason that the effective thickness depends on the function of frequency or not. As frequency goes higher, the difference increases. The effective thickness obtained from the fluid satisfying the Helmholtz equation is thinner than that of the fluid sat-

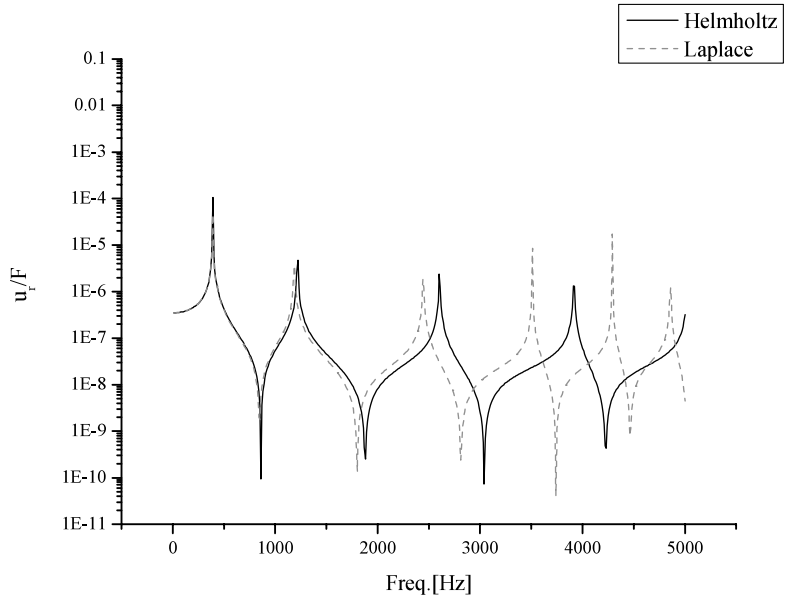

Fig. 6 Comparison of receptance assuming the internal fluid satisfying either Laplace's or Helmholtz's equation ( $n=$ 3)

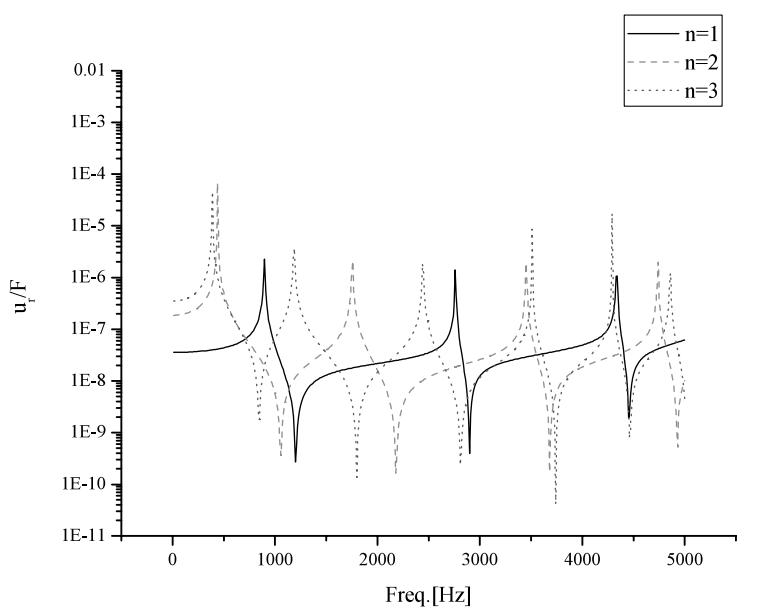

Fig. 7 Receptance according to the circumferential mode

isfying Laplace equation. Therefore, the mass, damping and stiffness obtained from Helmholtz equation is smaller than those obtained from the Laplace equation. It can be seen easily from Eq. (19) that the mass matrix increases and the stiffness matrix decreases due to the coupling effects of the internal fluid. Therefore, the effects of thinner thickness of the fluid makes less increasing of mass matrix and less decreasing of stiffness matrix. Therefore the resonance frequencies assuming fluid to satisfy Helmholtz equation are bigger than those assuming fluid to satisfy Laplace equation. Figure 7 shows the FRFs according the circumferential mode. The FRF of the shell given in Fig. 8 can be calculated by the complex summation of FRFs given in Fig. 7. The FRF at lower frequency shows a same tendency. But going higher frequency, the difference becomes bigger. In the Laplace equation, the effective thickness is overestimated comparing to the Helmholz equation.

Finally, the cylindrical shell with fluid flow is calculated. For calculation, the other test model is presented. This model has the following dimensions: length 


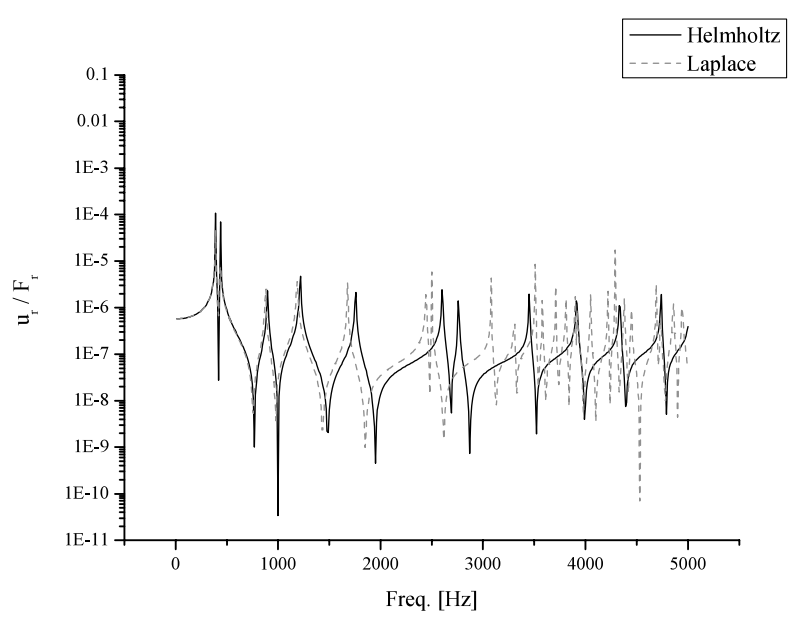

Fig. 8 Comparison of receptance of cylindrical shell

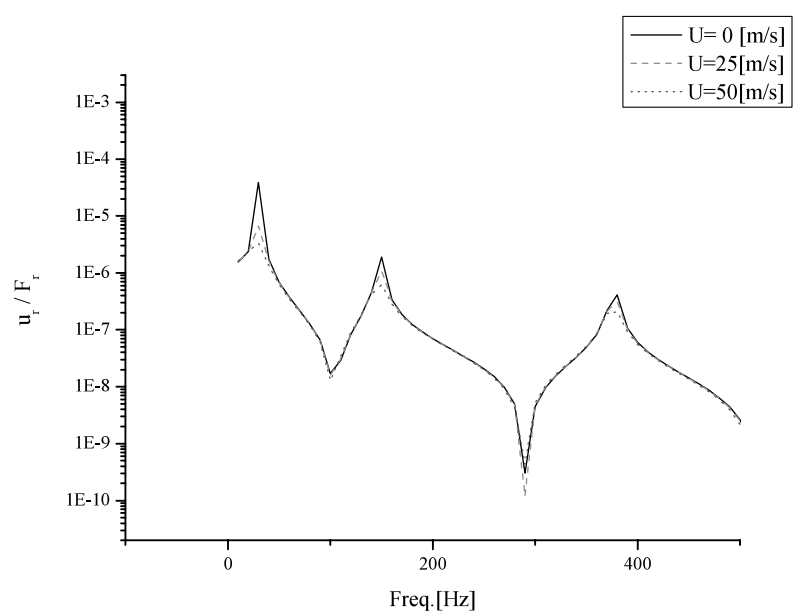

Fig. 9 The effects of mean velocity on receptance of the shell

$(L)=2[\mathrm{~m}]$, radius $(R)=0.1[\mathrm{~m}]$, thickness $(h)=0.002[\mathrm{~m}]$, Young's Modulus $(E)=2.1 \times 10^{11}\left[\mathrm{~N} / \mathrm{m}^{2}\right]$ and the others are the same as the previous case. The external radial force is applied at the end of the shell and receptance is obtained at the driving point.

Figure 9 shows the FRF according to the mean velocity. As the velocity increases, the resonance frequencies become lower and the damping effect increases.

\section{Conclusions}

(1) The finite element formulation of the shell with internal uniform flow was presented by assuming the internal fluid satisfies Helmholtz equation. The characteristics of the shell with the internal fluid satisfying Helmholtz equation was compared with the results with the fluid satisfying Laplace equation.

(2) The effective thickness varied by both the circumferential mode and the frequency, while the effective thickness of the fluid satisfying Laplace equation did not depend on the frequency.

(3) The effective thickness of the internal fluid ob- tained from the fluid satisfying Helmholtz equation was thinner than those obtained from the fluid satisfying Laplace equation.

( 4 ) The difference of the effective thickness between them became bigger as the frequency went higher.

( 5 ) The thinner thickness caused less increasing of mass effects and less decreasing of stiffness effects, which gave higher resonance frequencies.

(6) The effective thickness of the first circumferential mode was biggest and decreased more rapidly than those of higher modes as the frequency went higher.

(7) The velocity of the internal fluid had effects on the damping and stiffness of the cylindrical shell, which made the natural frequencies lower and peak values smaller.

\section{Acknowledgements}

The authors would like to thank the Ministry of Science and Technology of Korea for its financial support through a grant (M1-0230-00-0017) under the NRL (National Research Laboratory) project.

\section{References}

(1) Paidoussis, M.P. and Issid, N.T., Dynamic Stability of Pipes Conveying Fluid, Journal of Sound and Vibration, Vol.33 (1974), pp.267-294.

(2) Ginsberg, J.H., The Dynamic Stability of a Pipe Conveying a Pulsatile Flow, International Journal of Engineering Science, Vol.11 (1973), pp.1013-1024.

( 3 ) Chen, S.S. and Rosenberg, G.S., Free Vibrations of Fluid-Conveying Cylindrical Shells, Journal of Engineering for Industry, (1974), pp.420-426.

( 4 ) Mazuch, T., Horacek, J., Trnka, J. and Vesely, J., Natural Modes and Frequencies of a Thin Clamped-Free Steel Cylindrical Storage Tank Partially Filled with Water: FEM and Measurement, Journal of Sound and Vibration, Vol.193 (1996), pp.669-690.

( 5 ) Zhang, X.M., Liu, G.R. and Lam, K.Y., Coupled Vibration Analysis of Fluid-Filled Cylindrical Shells Using the Wave Propagation Approach, Applied Acoustics, Vol.62 (2001), pp.229-243.

( 6 ) Zhang, Y.L., Gorman, D.G. and Reese, J.M., A Finite Element Method for Modeling the Vibration of Initially Tensioned Thin-Walled Orthotropic Cylindrical Tubes Conveying Fluid, Journal of Sound and Vibration, Vol.245 (2001), pp.93-112.

( 7 ) Seo, Y.S., Jeong, W.B., Yoo, W.S. and Jeong, H.K., Frequency Response Analysis of Cylindrical Shells Conveying Fluid Using Finite Element Method, Journal of Mechanical Science and Technology, Vol.19, No.2 (2005), pp.625-633.

( 8 ) Petyt, M., Introduction to Finite Element Vibration Analysis, (1990), Cambridge University Press, Cambridge.

(9) Markus, S., The Mechanics of Vibrations of Cylindrical Shells, (1988), Elsevier, New York. 